Aus dem Pathologischen Institut der Universität in Berlin.

\title{
Ueber spontane Affentuberculose, ein Beitrag zur Tuberculosefrage.
}

\section{Von Dr. Lydia Rabinowitsch.}

Die Tuberculose der Affen ist eine alte, in Zoologischen Gärten wohl bekannte Erscheinung, darauf bezdgliche Angaben gehen bis zum Jahre 1843 zurück. So wurden z. B. nach verschiedenen Statistiken in London, Paris, Frankfurt a. M. Prozentzahlen bis zu 43 festgestellt. Anderseits erkranken Affen in ibrer Heimat auch nach den neueren Berichten von de Haan, v. Dungern, Bärmann und Halberstaedter selten spontan an Tubercalose.

Schon in seiner klassischen Tuberculose-Arbeit berichtet

R. Kochl) über zahlreiche Impfversuche mit Organmaterial und Reinkulturen von spontan an Tuberculose erkrankten Affen (8) auf verschiedene Laboratoriumstiere. Ebensolche mit Organmaterial hat auch $\mathrm{Straus}{ }^{2}$ ) angestellt, während Dieulaf oy und Krishaber ${ }^{3}$ ) erfolgreiche subcutane Verimpfungen von menschlichem tuberculösen

1) Die Aetiologie der Tuberculose (Mitteilungen aus dem Kaiserl. Gesundheits amt 1884, Bd. 2). - 2) La tuberculose et son bacille (Paris 1895). - 3) De lipoculation du tubercule sur le singe. (Arch. de Physiologie norm. et pathol. 1883, S. 484.) 
Material an Affen vorgenommen haben; gleichfalls positive Resultate erzielte $I m \mathrm{mach}^{1}$ ) durch Fütterung zweier Affen mit perlsuchtbacillenhaltiger Milch. Nach diesen Untersuchungen tring man keine Bedenken, die Tuberculose der Affen der Sãugetiertuberculose einzureihen, deren Erreger seinerzeit für identisch gehalten wurden.

Sehr bald nach dem Londoner Tuberculose-Kongreßs (1901), auf welchem sich Koch für die Nichtidentität der menschlichen und Rindertuberculose ausgesprochen hatte, wurden die Experimente am Affen von neuem aufgenommen, um an ihnen die Einwirkung der für verschiedene Tierspezies verschieden virulenten Menschen- und Rinderstämme in Erfahrung zu bringen und hieraus eventuelle Rückschlüsse auf die Infektionsmöglichkeit des Menschen mit Perlsucht zu ziehen.

Eine ganze Anzahl bezüglicher vergleichender Untersuchungen mit menschlicher und Rindertuberculose wurde vornehmlich an niederen Affenarten. nur eine einzige von Grünbaum an einem anthropoiden Affen (Schimpanse) vorgenommen. Yon der Mehrzahl der Autoren wurden neben orientierenden, subcutanen und intraperitonealen Impfungen Fütterungsversuche angestellt, da dieser Infektionsmodus $\mathrm{ja}$ auch bei der angenommenen natürlichen Uebertragung der Perlsucht auf den Menschen als der häufigste anzusehen ist.

Aus den Arbeiten von Grünbaum², Nocard ${ }^{3}$ ), de Jong'), Ravenel'b), de Schweinitz-Dorset-Schroeder ${ }^{6}$ ), de Haan ${ }^{7}$, de Gratias), Cipollina ${ }^{\circ}$ ), Beck ${ }^{10}$ ) nnd Macfadyen 11 ) geht nun unzweifelhaft hervor, daB gleich den menschlichen Tuberkel bacillen auch die Perlsuchtbacillen bei den Affen sowohl nach subcutaner und intraperitoncaler Infektion als nach Verfütterung Tuberculoze hervorzurufen vermögen. Bezüglich der Virulenzfrage ergaben die Infektionsversuche bei Affen ferner, daß nach mehrfachen Angaben bei den mit Perlsuchtbacillen geimpften Tieren stärkere und ausgedehntere tuberculöse Veränderungen zu verzeichnen waren. Diese Tatsache steht ja auch mit den an Kaninchen und Rindern vorgenommenen Virulenzprüfungen im Einklang, welche im allgemeinen eine größere Virulenz der Rinderstämme gegenüber den vom Menschen gewonnenen Tuberculosekulturen ergeben haben. Eine interessante Feststellung haben noch die Veısuche von Nocard wie auch die von de Schweinitz-Dorset-Schroeder gezeitigt, die entgegen der Angabe von de Gratia durch Affenpassage die Virulenz der Rindertuberkelbacillen herabsetzen konnten. Diese Tatsache würde im Verein mit weiteren experimentellen Ergebnissen der neueren Tuberculoseforschung auch die Virulenzabschwächung des Perlsuchtbacillus bei längerem Verweilen im menschlichen Korper nicht unwabrscheinlich machen.

Dieses waren unsere bisherigen Kenntnisse von der spontanen und experimentellen Affentuberculose, als ich im Dezember 1903 an die wissenschaftliche Bearbeitung des aus dem Berliner Zoologischen Garten stammenden Affenmaterials heranging, welches mir Herr Professor Heck und Dr. Heinroth in liebensw ürdigster Bereitwilligkeit zur Verfügung stellten. Für die Ueberlassung einer ganzen Anzahl toter Affen bin ich ferner meinem hochverehrten Gönner, Herrn Professor Lassar. $\mathrm{zu}$ herzlichem Dank verpflichtet.

Alle die bisherigen, nach dem Londoner Vortrage Kochs an Affen angestellten Versuche hatten sich, wie wir gesehen, lediglich mit der Frage beschäftigt, ob auch dieser dem Menschen anatomisch nahe verwandten Tierspezies eine verschiedene Empfänglichkeit für die einzelnen Tuberculoseformen zu eigen ist. Es ist aber bis jetzt keiner der Untersucher an die Frage herangetreten, ob sich wie beim Menschen auch bei den in der Gefangenschaft spontan an Tuberculose erkrankten Affen mit verschiedener Virulenz begabte Tuberculosestämme vorfinden, die eine Trennung in verschiedene Varietäten zulassen und somit einen Rückschluß auf die Herkunft der betreffenden Stämme gestatten. Denn die experimentelle Fest-

1) Report on the transmissibility of bovine tuberculosis through milk to young ani mals. (British Medical Journal, July 26 1884.) - 2) Die Uebertragbarkeit der Perlsucht au Affen. (Verhandiungen der Tuberculose-Kommission der Gesellschaft Deutscher Naturforscher und Aerzte, Hamburg, 1901, Berlin, Hirschwald, 1902.) - 3) Presse Vétérinaire, 1902, Avril, Mai, Novemhre. - 4) Semaine Médicale, 15. Jan. 1902. - 5) The inter. communicability of human and bovine tuherculosis (University of Pennsylvania Medical Bulletin, May 1902). -- 6) Virulence of the bovine tuberculosis for monkeys (American callo Medicine, January 4, 1902). Experiments concerning tuberculosis Part 1 , S. 66. Experiments upon monkeys. (U. S. Department of Agriculture, Bureau of Animal Industry, Bulletin No. 52, 1905.) - 7) Experimentelle Tuberculose beim Affen. (Fortschritte de Veterinärhygiene 1903, H. 4 und Virchows Archiv 1903, Bd. 174, S. 1.) - 8) XI. Congrès International d'Hyglène et de Démographie, Bruxelles Septembre 1903 - 9) Beitrag zu dem Studium der Rinder- und menschlichen Tuberculose (Berliner kllnische Wochenschrift 1903, No. 8). - 10) Beitrăge über die Unterscheldung der Bacillen von mensch licller und tierischer Tuberculose, namentlich nach Infektion verschiedener Tiere. (Fest schrift für Robert Koch. Fischer, Jena 1903, S. 611.) - 11) Upon the virulence of the bacillus of bovine and human tuberculosis for monkeys. (The Lancet, Sepl. 12. 1903 , S. 745.) stellung der Uebertragbarkeit von Tuberculosestämmen verschiedener Herkunft auf Affen beweist noch nicht die allerdings nach unseren Erfahrungen sehr naheliegende Tatsache, daB Affen auch spontan bei gegebener Gelegenheit sich mit den verschiedenen Tuberculoseerregern infizieren können.

Zur Entscheidung dieser Frage war das Material des hiesigen Zoologischen Gartens besonders geeignet. da in demselben den Affen sowohl Gelegenbeit zur Infektion mit den verschiedenen Formen der Säugetiertuberculose wie mit Geflügeltuberculose geboten ist. Es wurden von mir im ganzen 45 tuberculöse Affen untersucht. Unter diesen befanden sich 1 Orang, 1 Schimpanse, 1 Dschelada, 1 Hutaffe, 1 Kapuziner, 1 Husarenaffe, 1 Pinseläfchen; ferner 3 Paviane. 6 Mangaben, 5 Javaaffen, 5 Makaken, 9 Rhesusaffen und 10 Meerkatzen

Es waren also die verschiedensten Affenarten, welchen zum Teil in Einzelkäfigen, meistenteils aber in gemeinschaftlichen Käfigen sowohl die Möglichkeit zur Infektion von außen wie zur gegenseitigen Ansteckung geboten war. Es mag hier noch hervorgehoben werden, dab ich aufier den tuberculösen Tieren noch eine große Anzahl jüngerer und älterer Affen. besonders auch aus den gemeinschaftlichen Käfigen, untersucht habe, die keine Tuberculose aufwiesen Diese Tatsache beweist uns, daf ebenso wie beim Menschen auch beim Affen außer dem Tuberkelbacillus noch eine besondere Disposition zum Zustandekommen einer tuberculösen Erkrankung erforderlich ist.

Was das pathologisch-anatomische Bild bei der spontanen Affentuberculose betrifft, so hat $R$. Koch schon in seiner klassischen Tuberculosearbeit darauf hingewiesen, daß die Tuberculose der Affen von der des Menschen in mehrfacher Beziehung verschieden ist.

"Sie bleibt gewöhnlich nicht lange auf ein Organ beschränkt, sondern verbreitet sich frühzeitig über den ganzen Körper. Dann tritt sie aber nicht. wie die menschliche Miliartuberculose, in Form von zahlreichen Knötchen auf, welche eine gleichmäßige Größe besitzen. sondern führt zur Bildung einer größeren oder geringeren Anzahl von tuberculósen Herden, welche sehr verschieden groß sind. und besonders in der Leber, Milz und Drüsen anstatt der festen. käsigen Substanz der tuberculósen Herde des Menschen ziemlich dünnflussigen Fiter enthalten, sodaß sie eher den Eindruck von multiplen Abscessen als von Tuberkeln machen. Daneben kommen allerdings auch die typischen Formen des grauen Tuberkels mit gelblichem Zcntrum in der Lunge, auf der Pleura und dem Netz vor. Aber auch diese sind von sehr verschiedener Größe, und man gewinnt den Eindruck, als ob beim Affen die Ausbreitung des Tuberkelvirus nicht auf einmal, wie bei der menschlichen Milıartuberculose, sondern kontinuierlich und nur in geringen Mengen vor sich geht."

R. Koch nahm an, dab der erste Infektionsherd bei den Affen meistens in der Lunge sich befindet. Bei den von mir untersuchten Affen. die eigentlich fast alle Stadien der Tuberculose aufwiesen, waren nur fünfmal die Brustorgane allein befallen, neunmal lag eine Tuberculose der Bauchorgane und $31 \mathrm{mal}$ eine solche der Bauch- und Brustorgane vor. Nach unseren heutigen Erfahrungen und divergierenden Anschauungen über die verschiedenen Infektionswege bei der Tuberculose wäre es mübig, auf Grund der Sektionsergebnisse den einen oder andern Infektionsmodus bei der Affentuberculose in den Vordergrund stellen zu wollen. Nach den obigen Zahlen könnte es ja beinahe scheinen, als ob der bei der menschlichen Tuberculose durch v. Behring wiederum hervorgehobene und zur Anerkennung gelangende Infektionsmodus der Fütterungstuberculose bei den Affen eine gröbere Rolle spielt als die Inhalationstuberculose, gleichgültig, ob es sich um Tuberkelbacillen tierischer oder menschlicher Herkunft handelt.

Der größte Teil der von mir untersuchten Affen gelangte ziemlich bald nach dem Tode zur Sektion.

In einer Anzahl von Fällen war Dr. Max Koch, bisheriger Assistent am Pathologischen Institut und jetziger Prosektor in Elberfeld, im AnschluB an unsere gemeinschaftlichen Tuberculose. arbeiten so freundlich, die Sektionen auszuführen. Das Sektionsprotokoll des einen, von Prof. Lassar stammenden Schimpansen verdanke ich Herrn Prof. O. Israel. Die Protokolle sowie die näheren Angaben über die ausgeführten Versuche sollen in der ausführlichen Arbeit mitgeteilt werden. An dieser Stelle möchte ich mich auf eine Zusammenfassung derselben beschräuken und eine Besprechuıı Jer Versuchsergebnisie anreihen. 
Von den tuberculösen Organen wurden stets mikroskopische Ausstrichpräparate angefertigt, in denen sich die Tuberkelbacillen im Gegensatz zu menschlichem Material meistenteils ohne Begleitbakterien vorfanden. Mit dem Ausgangsmaterial wurden nur von 15 Affen zum Teil mit verschiedenen Organen Uebertragungsversuche an Meerschweinchen und in geringerer Zahi an Kaninchen angestellt. Die Kulturen wurden teils von diesen mit dem Ausgangsmaterial geimpften Tieren gewonnen, zum größten Teil jedoch wurden sie direkt aus den tuberculösen Affenorganen isoliert, was bei dem seltenen, bereits erwähnten Vorkommen von Begleitbakterien relativ leichter gelang als bei der Züchtung menschlicher Tuberculosekulturen. Bezüglich der Gewinnung der Kulturen verweise ich auf meine soeben erschienene Publikation: "Untersuchungen ïber die Beziehungen zwischen der Tuberculose des Menschen und der Tiere. "1)

Von den tó tuberculösen Affeu wurden von 33 Tieren im ganzen 43 Kulturen gezuchtet und diese bezuglich Wachstum, kultureller Eigenschaften und Tierpathogenität geprüft. Bezïglich der Methodık dieser verschiedenen Prüfungen verweise ich wiederum auf meine eben zitierte Arbeit. Mit abgewogenen Mengen der isolierten Reinkulturen wurden neben Meerschweinchen vornehmlich Kaninchen geimpft, da diese Tierspezies nach den neueren experimentellen Erfahrungen gleich den Rindern die geeignetste ist. die Virulenz verschiedener Tuberculosestämme festzustellen und auf Grund dieser Virulenzprïfung im Verein mit den Tachstumseigenschaften der betreffenden Kultur auf ihre Herkunft, ob Menschen- oder Rinderstamm. zu schließen. In einigen Fällen, in denen die isolierte Tuberculosekultur den Verdacht eines Geflügeltuberculosestammes wach rief, wurden natirlich Versuche an Hühnern angeschlossen.

Von den genannten 33 eingehend untersuchten tuberculösen Affen ( 43 Kulturstämme) wurden von 27 Tieren $3 \pm$ Stämme kulturell und im Tierversuch geprüft, von 6 Affen 9 Kulturen nur durch das Kulturverfahren. Das Ergebnıs dieser vergleichenden Untersuchungen war kurz folgendes: Lnter den 27 tuberculösen Affen fanden sich

in 19 Fällen menschliche Tuberculosestämme.

" 3 Fällen Rinderstämme,

" 1 Fall in der Lunge ein menschlicher, in der Milz ein Rindertuberculosestamm,

. 2 Fällen L'ebergangsformen von menschlicher und Rindertuberculose,

, 1 Fall Geflügeltuberculose,

. 1 Fall Uebergangsform von Geflügel- und menschlicher Tuberculose.

Lie 9 von 6 Affen stammenden und nur bulturell untersuchten Tuberculosekulturen konnten als menschliche Stämme bezeichnet werden.

Bezüglich der Unterscheidung smerkmale der menschlichen Tuberkelbacillen von den Rinderbacillen soll hier nur erwähnt werden, daß die Unterschiede vornehmlich in dem kulturellen Verhalten bestehen, insofern die Menschenstämme sich leichter und üppiger züchten lassen, und in der größeren Virulenz der Rinderstämme für Kaninchen. Zu bemerken ist ferner, dab im allgemeinen ein Zusammenhang von Wachstums- und Virulenzeigenschaften zu konstatieren ist, indem sich virulentere Stämme schwieriger kultivieren lassen und ein spärlicheres Wachstum zeigen als leichter zu gewinnende und üppiger wachsende Kulturen von geringerer Virulenz. Zwischen den Erregern der Geflügeltuberculose und denen der Säugetiertuberculose (menschliche und Rinderbacillen) ist auBer ausgesprochenen kulturellen Unterschieden (Aussehen und Wachstum) besonders die hohe Pathogenität der Geflügeltuberculosebacillen für Hühner hervorzuheben. Als Uebergangsformen endlich der menschlichen, Rinder- und Geflügeltuberculose sind solche Stämme zu betrachten, die sich bezüglich ihres Gesamtverhaltens nicht in eine Kategorie einreihen lassen, sondern die hinsichtlich ihrer kulturellen Eigenschaften der einen Form, hinsichtlich ihrer Tierpathogenität einer andern anzugliedern sind.

In der Mehrzahl unserer Fälle von Affentuberculose konnten demnach menschliche Tuberculosekul-

1) Arbeiten aus dern Pathologischen Instıtut in Berlin. Berlin, Hirschwald, 1906, 5. $365-436$ turen, in einer relativkleinen Anzahl Rinderkulturen, in einem Fall beide vergesellschaftet und in einem andern Fall eine Geflügeltuberculosekultur gewonnen werden. In einigen weiteren Fällen wurden sogenannte Uebergangsformen oder atypische Stämme is oliert.

Eine Klassifizierung der bei der spontanen Affentuberculose gefundenen Tuberculoseerreger war, wie eingangs der Arbeit erwähnt, in früherer Zeit nicht vorgenommen worden. Nur in ver'schiedenen Arbeiten von $\mathrm{Hu}$ eppe-Fischel finden wir die kurze Angabe, daß sich bei Affentuberculose Bacillen züchten ließen, die in ihrer Wachstumsform von jener der typischen Hühnertuberculose nicht unterschieden werden konnten. Scheinbal sind aber diese von $\mathrm{Fischel}^{1}$ ) gewonnenen Kulturen nicht auf ihre Pathogenitȧt für Hühner geprüt worden

Aus der Beschreibung der von $R$. Koch ausgeführten Tierversuche mit Reinkulturen von Affentuberculose (Stamm 11) geht ferner hervor, daß es sich in diesem Fall aller Wahrscheinlichkeit nach um Rindertuberkelbacillen gehandelt hat.

Diese meine Vermutung glaube ich durch folgende Bemerkung Kleines $\left.{ }^{2}\right)$ in seiner soeben erschienenen Mitteilung viber Impftuberculose durch Perlsuchtbacillen bestatigt zu finden: „Einer der ältesten Perlsuchtstämme der Sammlung des Instituts wurde von R. Koch vor etwa 20 Jahren aus einem Affen gezüchtet.

Auch die von Dammann und Müssemeier ${ }^{3}$ ) isolierte und geprüfte Affenkultur scheint nach ihrer hohen Virulenz für Kaninchen den Rinderstämmen zugerechnet werden zu müssen.

Eine Prädilektion der verschiedenen Tuberculoseerreger für bestimmte Organe möchte ich nach den vorliegenden Protokollen meiner Fälle von Affentuberculose und nach den bisherigen Untersuchungen über die Beziehungen zwischen der menschlichen und tierischen Tuberculose kaum annehmen, obwohl gerade in zweien meiner drei Fälle von Rinderbacillenbefunden lediglich eine Tuberculose der Bauchorgane konstatiert wurde, da anderseits in mehreren Fällen von Abdominaltuberculose typische menschliche Stämme isoliert werden konnten. Gegen eine etwaige Annahme, daß die Rindertuberkelbacillen vermöge besonderer biologischer Eigenschaften eine größere Affinität zu dem Verdauungstraktus der Affen als zu ihrem Respirationstraktus besitzen, sprechen auch die bei der experimentellen Affentuberculose erhobenen Befunde. So konnten Ravenel, de Schweinitz-Dorset-Schröder und Macfadyen bei Affen durch Verfütterung von Rinderbacillen eine Tuberculose der Lungen mit Freibleiben des Darmes hervorrufen, während Macfadyen bei Verfütterung von tuberculösem Sputum in sämtlichen positiven Uebertragungsversuchen tuberculöse Lrarmgeschwüre erzeugte. Um ein gesetzmäßiges Verhalten dürfte es sich aber weder in der einen noch in der andern Richtung handeln, da anderseits de Gratia bei seinen zahlreichen Fütterungsversuchen an Affen mit Rinderund Schweinetuberculose sowie mit perlsuchtbacillenhaltiger Milch meistenteils eine Mesenterialdrüsen- und Darmtuberculose unter sekundärer Beteiligung der Lungen hervorrief. Auch von Dungern ${ }^{4}$, dessen Arbeit nach dem vorläufigen $\mathrm{Ab}$ schluB meiner Affenversuche erschienen ist und auf die ich noch weiter unten zurückkommen werde, konnte im Gegensatz zu den oben genannten Autoren bei zwei mit Perlsuchtbacillen gefütterten anthropoiden Affen IGibbons, Hylobates, welche nach dem Catalogus mammalium tam viventium quam fossilium, Trouessart, letzte Auflage 1904, zu der dem Menschen am nächsten stehenden Affenfamilie Simiidae zu rechnen sind) eine typische Intestinaltuberculose feststellen, während bei zwei Gibbons nach Verfütterung von menschlichen Kulturen primäre Lungentuberculose zu konstatieren war.

Ebenso wie meine Befunde verschiedener Tuberculoseerreger bei spontaner Affentuberculose eine Beziehung zur Eintrittspforte, respektive zur Lokalisation der tuberculösen Erkrankung vermissen lieben, ebensowenig waren irgendwelche Beziehungen zwischen der Schwere der im Affenkörper ge-

1) Untersuchungen über die Morphologie und Biologie des Tuberculose erregers. (Wien, Braumüller 1893 und Berliner Klinische Wochenschrift 1893, No. 41) 2) Zeitschrift für Hygiene, Bd. 52, 1906, S. 512. - 3) Untersuchungen über die Bezlehungen zwischen der Tuberculose des Menschen und der Tiere. (Hannover, Schaper 1905) - 4) Beitrag zur Tuberculosefrage auf Grund experimenteller Untersuchungen an anthropoiden Affen (Münchener Mędizinische Woçhenschıift 1906, No. 1) 
setzten tuberculösen Veränderungen und der durch den Tierversuch festgestellten Virulenz der isolierten Kulturen aufzufinden, wie dies ja auch nach den neueren experimentellen Feststellungen bei der menschlichen und der Rindertuberculose der Fall ist. Da wir anderseits aber in nicht unberechtigter Weise aus dem Wachstum und der Virulenz der geprüften Kulturen auf die Herkunft der Stämme zu schlieben pflegen, so haben unsere zum erstenmal systematisch bei einer gröBeren Anzahl tuberculöser Affen durchgeführten Untersuchungen ergeben, dab die Affen in der Gefangenschaft sich mit den verschiedenen Tuberculoseerregern je nach gegebener Gelegenheit infizieren können.

$\mathrm{DaB}$ die Gelegenheit zur Infektion mit menschlichen Tuberkelbacillen naturgemäßerweise bei den Affen die bei weitem größte ist, da mit der Nahrung eingeführte Perlsuchtbacillen wenig in Frage kommen dürften, haben meine zahlenmäßigen Angaben bestätigt. Es ist ferner leicht verständlich, daß in einem zoologischen Garten auch Gelegenheit zur Infektion mit Geflügeltuberculosebacillen geboten ist. Meine Befunde von Uebergangsformen bei spontaner Affentuberculose Iehren des weiteren, daß auch in diesem Wirtstier ebenso wie im menschlichen Körper eine Umwandlung der einen Tuberculoseform in eine andere nicht ausgeschlossen erscheint. Die Gründe, welche eine derartige Annahme berechtigt erscheinen lassen, habe ich in meiner oben zitierten Arbeit ausführlicher dargetan. Diese Annahme wird gestützt durch die bereits erwähnten Resultate von Nocard, de Schweinitz-DorsetSchroeder, welche durch Affenpassage die Virulenz der Rindertuberkelbacillen herabsetzen konnten.

Gleich den früheren Autoren, welche vergleichende Uebertragungsversuche an niederen Affen angestellt und mitunter eine größere Empfänglichkeit derselben für Rinder- als für menschliche Bacillen konstatiert hatten, verfolgte auch v. Dungern durch seine Virulenzprüfungen an anthropoiden Affen den Zweck, aus ihrem Ergebnis Rückschlüsse auf die Infektionsmöglichkeit des Menschen mit Perlsucht zu ziehen.

"Diese Tiere stehen dem Menschen ja ganz besonders nahe“, sagt $v$. Dungern, ndafür spricht nicht nur die weitgehende anatomische Uebereinstimmung, sondern auch die gleichartige Präzipitinreaktion und das ähnliche Verhalten manchen spezifisch menschlichen Infektionsk rankheiten gegenüber [Typhus, Pocken, Syphilis] “. ${ }^{1}$ )

Da v. Dungern nun sowohl bei seinen quantitativen Virulenzprüfungen durch subcutane Infektion wie bei den Fütterungsversuchen einen Unterschied in der Wirkung der Perlsuchtbacillen und der menschlichen Tuberkelbacillen in keiner Weise konstatieren konnte, so liegt seines Erachtens kein Grund vor, eine verhältnismäßig geringe Virulenz der Perlsuchtbacillen für den Menschen anzunehmen, sondern es müsse auf Grund dieser Untersuchungen prinzipiell an der Gefährlichkeit der Rindertuberculose für den Menschen festgehalten werden. Diese logische Schlußfolgerung v. Dungerns ist weder überraschend noch unberechtigt, wie Kleine in seiner Kritik der v. Dungernschen Versuche bemerkt, sondern sie stützt sich eben auf die Tatsache, daß die anthropoiden Affen dem Menschen doch außerordentlich viel näher stehen als dem Rinde, auch was die Empfänglichkeit für Infektionserreger betrifft. Wenn es überhaupt erlaubt ist, aus der für einzelne Tierspecies festgestellten Virulenz verschiedener Tuberculosestämme Schlüsse auf die Virulenz beim Menschen zu ziehen, dann dürften jedenfalls, entgegen der Annahme Kleines, die Versuche an höher stehenden Affen eher für den Menschen zu verwerten sein als die an niederen Affen oder gar an Kaninchen und Rindern ausgeführten Uobertragungsversuche.

Zur Entscheidung der uns interessierenden Frage können wir jedoch nunmehr dieser indirekten Beweisführung entraten, so dankenswert jene experimentellen Feststellungen an Affen auch waren, seitdem von der Mehrzahl der Autoren wie auch vom Kaiserlichen Gesundheitsamt in Berlin auf Grund von Perlsuchtbacillenbefunden beim Menschen die Infektionsmöglichkeit desselben durch die Rindertuberculose anerkannt worden ist. Ebenso wie der Mensch je nach gegebener Infektions-

1) Der Schimpanse leidet auch an Anchylostoma duodenale. gelegenheit für die verschiedenen Tuberculoseerreger empfänglich sein kann, in gleicher Weise können sich nach meinen Untersuchungen sowohl niedere wie höhere Affen mit ihnen auch spontan infizieren. Diese Infektionsmöglichkeit der Affen mit menschlichen und Rinderbacillen steht demnach vollkommen in Einklang mit den Ergebnissen v. Dungerns und denen früherer Autoren bei künstlicher Infektion.

Uebrigens sind nach neueren Untersuchungen über experimentelle Hauttuberculose der Affen von Kraus und Kren ${ }^{1)}$ sowie Baermann und Halberstädter ${ }^{2}$ ) [Java-Expedition von Neisser] diese Tiere auch für die cutane Infektion leicht empfänglich. Die fortgesetzten vergleichenden Versuche dieser Autoren ${ }^{3}$ ) mit verschiedenen Tuberculosestämmen werden voraussichtlich ebenfalls $\mathrm{zu}$ einem positiven Resultate führen, ebenso wie auch der Mensch eine Hauttuberculose sowohl durch Infektion mit menschlichem wie Perlsuchtmaterial akquirieren kann. Ja ich glaube, daß beim Affen auch mittels Geflügeltuberculosebacillen eine experimentelle Hauttuberculose zu erzeugen sein wird, da ja z. B. auch bei Vögeln und speziell bei den häufig mit menschlicher Tuberculose spontan infizierten Papageien eine solche sowohl mit Bacillen der Säugetier- wie Hühnertuberculose zu erzielen ist, obwohl Héricourt und $R i c h e t^{4}$ ) schon vor Jahren über zwei negative subcutane Uebertragungsversuche des type aviaire auf Affen berichtet hatten. In dieser Voraussetzung habe ich Herrn Geheimrat $\mathrm{Ne}$ isser, dem ich auf seinen Wunsch gern eine Anzahl verschiedener Säugetiertuberculosestämnie für die von seinen Assistenten in Batavia fortgesetzten Versuche überlieB, auch einige Geflügeltuberculosekulturen zur Verfügung gestellt, da ich selber zur Ausführung derartiger Experimente bisher nicht in der Lage war.

Wenn ich mich schon auf Grund meiner Beobachtungen über die Tuberculose der Vögel ") für berechtigt hielt, die Erreger der Geflügel- und Säugetiertuberculose als verschiedenen Tierspezies angepaßte Varietäten einer Grundform aufzufassen, so haben mich meine weiteren Tuberculosestudien wie auch die an Affen vorgenommenen Untersuchungen in meiner Ueberzeugung von der Arteinheit des Tuberkelbacillus noch mehr gefestigt. Wir dürfen uns bei Erörterung der Identitätsfrage nicht einseitig an die morphologischen und biologischen sowie tierpathogenen Eigenschaften der aus dem tuberculoseinfizierten Organismus gezüchteten Tuberculoseerreger klammern, sondern müssen außer diesen, zum Teil variablen Größen und speziell den Virulenzschwankungen auch die verschiedene Natur- und Krankheitsanlage der einzelnen Tierspezies wie die Reaktionsverschiedenheit der tierischen Gewebe ins Auge fassen und miteinander in Beziehung bringen. Unter Berücksichtigung all dieser Momente dürfte eine synthetische Auffassung, wie ich sie mit meiner unistischen Anschauung vertrete, zumal vom praktischen, hygienischen Standpunkt aus, nicht unberechtigt erscheinen.

1) Ueber experimentelle Erzeugung von Hauttuberculose bei Affen. Sitzungsbericht der Kaiserlichen Akademie der Wissenschaften in Wien Bd, 114, Abtell. III, bericht der Kain Wochenschrift 1906 , No. 7 .

3) Kraus berichtet in der K. K Gesellschaft der Aerzte in Wien (Munchener medizinische Wochenschrift 1906, No. 1, S. 49), daB er sowohl mit Kulturen des Typus humanus wie mit solchen des Typus bovinus tuberculöse Hauterkrankungen bei Alfen erzeugte, die der menschlichen Tuberculose ähnlich sahen.

4) De l'état réfractaire du singe à la tuberculose aviaire. Comptes rendus de la société de biologie 1891, S 802 . 5) Die Geflügeltuberculose und ihre Beziehungen zur Sãugetiertuberclusoe. Deutsche medizinische Wochenschrift 1904, No. 46. 